\title{
The science of complexity and the role of mathematics
}

\author{
T. Bountis ${ }^{1, a}$, J. Johnson ${ }^{2}$, A. Provata ${ }^{3}$, and G. Tsironis ${ }^{4}$ \\ 1 Center for Research and Applications of Nonlinear Systems Department of Mathematics \\ University of Patras, Patras, Greece \\ 2 British Open University, Milton Keynes, UK \\ 3 Institute of Nanoscience and Nanotechnology, National Center for Scientific Research \\ "Demokritos", 15310 Athens, Greece \\ 4 Department of Physics, University of Crete, Heraklion, Greece
}

Received 28 March 2016 / Received in final form 26 July 2016

Published online 30 September 2016

\begin{abstract}
In the middle of the second decade of the 21st century, Complexity Science has reached a turning point. Its rapid advancement over the last 30 years has led to remarkable new concepts, methods and techniques, whose applications to complex systems of the physical, biological and social sciences has produced a great number of exciting results. The approach has so far depended almost exclusively on the solution of a wide variety of mathematical models by sophisticated numerical techniques and extensive simulations that have inspired a new generation of researchers interested in complex systems. Still, the impact of Complexity beyond the natural sciences, its applications to Medicine, Technology, Economics, Society and Policy are only now beginning to be explored. Furthermore, its basic principles and methods have so far remained within the realm of high level research institutions, out of reach of society's urgent need for practical applications. To address these issues, evaluate the current situation and bring Complexity Science closer to university students, a series of Ph.D. Schools on Mathematical Modeling of Complex Systems was launched, starting in July 2011 at the University of Patras, Greece (see http://www. math.upatras.gr/ phdsch11). These Schools lasted two weeks each and included, beyond introductory lectures, a conference component of 2-3 days where students were exposed to recent results mainly presented by young researchers. The Ph.D. Schools continued successfully, the 2nd one taking place at Pescara, Italy (2012), (see http://www.nodycosy.unich.it), the 3d one at Heraklion, Crete, Greece (2013) (see http://nlsconf2013. physics.uoc.gr) and the 4th one in Athens, Greece (2014) (see http://nlsconf2014.physics.uoc.gr) (2014). This special theme issue is based on the introductory lectures and research talks presented at the 5th Ph.D. School-Conference of this series held at the University of Patras, Greece, 20-30 July, 2015 (see http:// www.math. upatras.gr/ phdsch15). The primary concern of all those
\end{abstract}

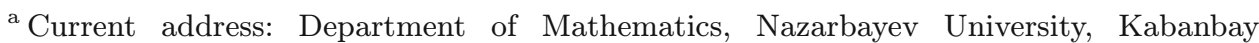
batyr 53, 010000 Astana, Republic of Kazhakstan.
} 
that participated in these events was to emphasize the role of mathematics, modeling and numerical simulation, which are indispensable for understanding what we call complex behavior of physical, biological, technological and socio - economical systems. In the discussions that took place, a great number of participants expressed the need to formulate a unifying theory of complex systems based on the main conclusions that have been reached so far in the science of Complexity. As Guest Editors of this volume, we also feel that it is important to reach some fundamental conclusions concerning common phenomena, theories and methodologies that arise in Complexity. We should all work to explore common rules and approaches, particularly in view of the remarkable challenges that face us all regarding complex social problems that threaten present day society and civilization as we know them.

\section{Introduction}

"Complexity" is the latin version of the Greek word $\pi \circ \lambda u \pi \lambda$ oкó $\tau \zeta$, which refers to a multitude of twisting and folding structures similar to what one finds in the braids of a lady's hair, the foliages of a tree or the flocking behavior of birds. At first sight, an object, or natural phenomenon characterized as complex (or "polyplokon") evokes feelings of confusion and perplexity. When expressed in mathematical terms, however, it often reveals deep geometrical, dynamical and statistical properties and global unifying features that allow us to associate it with some particular universality class. This type of increased understanding of a complex system's behavior is achieved by the discovery of appropriate mathematical models.

In the past four decades, it has become abundantly clear that complex behavior does not require an infinite (or even very large) number of variables to manifest itself. Indeed, determinism and predictability break down already at the level of very few dimensions, leading to a wide variety of simple nonlinear models, which are characterized by classical chaos, but may also produce very complicated phenomena when viewed in the framework of quantum mechanics.

On the other hand, if we wish to tackle problems involving many variables, we need to understand the behavior of the system as a whole. In this regard, we are no longer interested in the trajectories of individual particles, but wish to analyze the statistical behavior of the particular ensemble. We thus discover that the most interesting systems of natural, biological and social sciences are far from equilibrium, and exhibit self organization and emergence of patterns and coherent structures that cannot be explained by the behaviour of individual components. These are known as complex systems.

Complexity finds its origins in Physics, Chemistry, Biology, Computer Science and Engineering, but has spread by now in the realms of Medicine, Economics and the Social Sciences. Its merits and promises had already been foreseen in a number of volumes of the $1980 \mathrm{~s}$ and 1990 s (see e.g. [1,2]), but it was not until the 21 st century that its importance as a new science was widely recognized. The surprising realization that complex systems are governed by scaling laws and "universal" principles makes it plausible that mathematical modeling can be used to help us understand them better and hopefully uncover some of their mysteries [3].

The present special theme issue of the European Physics Journal (EPJ ST) aims to give a glimpse of recent progress in using mathematical models to probe more deeply into a wide variety of complex phenomena occurring in practically all sciences. It is based on lectures, talks and selected posters presented at the 5th Ph.D. School on "Mathematical Modeling of Complex Systems" that took place at the University 
of Patras, Patras, Greece, 20-30 July, 2015 (http://www.math.upatras.gr/ $\sim$ phdsch15). The main idea behind this event follows a similar rationale adopted more than 10 years ago in another International Conference dedicated to Complexity, also organized in Patras in July 2004, whose proceedings appeared as two special issues of the International Journal of Bifurcation and Chaos in 2006 [4]. Accordingly, 4 more Ph.D. Schools of the same format were organized on the topics of complexity and mathematical modelling: 2011 in Patras, 2012 in Pescara, Italy, 2013 at Heraklion, Crete and 2014 in Athens.

\section{The reviews section}

The 5th Ph.D. School of Patras was attended by nearly 60 graduate students, 25 of whom were Greek, and 35 came from other European countries, as well as Iran, Kazakhstan, Mexico, China and Japan. Since the main purpose of the Ph.D. School was educational, there were several review lectures emphasizing mathematical models used in multidisciplinary topics of many sciences. We thus decided in this EPJ ST to start with a Review Section containing papers written by some of the main lecturers of the School. The first one is a review by Eckehard Schöll on the interplay between topology and dynamics to understand synchronization phenomena in complex networks of oscillators $[5,6]$ such as the surprising coexistence of synchronous and asynchronous populations (so called chimera states) in systems governed by FitzHugh-Nagumo, Van der Pol, or Stuart-Landau dynamics.

The lecture by Manuel G. Velarde focuses on many years of research by his group on the possibility of controlling long range electron transport in crystalline solids using mathematical ideas of nonlinear wave propagation, solitons and discrete breather theory. In this regard, it is useful to read the next review by Vassilis Rothos describing some fundamental analytical and numerical approaches to the study of propagation of nonlinear waves, their stability and bifurcation properties in discrete and continuous mathematical models of the nonlinear Schrödinger type.

This is followed by a review written by Marko Robnik on the fundamental ideas and methods of the field of quantum chaos, dealing with simple models that possess regular and chaotic eigenstates and emphasizing the importance of quantum localization which affects the statistical distributions of energy level spacings. Next, Loukas Vlahos and Heinz Isliker report on the use of tools of complexity to understand turbulent convection in the solar atmosphere. They analyze the fractal properties of emerging magnetic flux structures and study the fragmentation of these structures using cellular automata and the theory of self organized criticality. This is followed by the review of Kyriaki Antoniadou, who focuses on recent applications of classical methods of Celestial Mechanics to the analysis of periodic orbits, resonances and stability in systems of few celestial bodies. These studies help us better understand the dynamics of exosolar planetary systems that are discovered almost daily 50-100 light years away from our solar system.

Finally, Tassos Bountis emphasizes in his review the importance of long range interactions joining the nodes of nonlinear oscillator networks. In particular, if these nodes are particles of a mechanical system, the range of their interactions strongly affects their global dynamics and statistics, allowing us to distinguish between weak and strong chaos and Boltzmann Gibbs or Tsallis thermostatistics [7,8]. On the other hand, if the nodes model neuron oscillators, sufficiently long range interactions can lead to the appearance of these intriguing chimera states also discussed in the review by E. Schöll. 
The present EPJ ST volume continues with two more sections of contributions listed as Papers I and Papers II, containing respectively articles that are partly reviews and partly new material in Sect. I, and almost exclusively new results in Sect. II.

\section{The section of papers I}

Section I begins with a paper by J. Johnson entitled "Hypernetworks: multidimensional relationships in multilevel systems". In this work, the author draws our attention to the fact that, despite the great importance attributed today to networks as models of complex systems, little attention has been paid to classes of networks that have multiple (or n-ary as he calls them) relations between their vertices. Simplicial families, hypersimplices and hypernetworks are introduced, with multidimensional connectivity structure designed to study the complex dynamics of interacting multilevel systems. These represent natural generalizations of networks in which multilevel operations can be defined with rich mathematical properties that can prove useful in many applications.

The section continues with an analytical study of chaos, by G. Contopoulos et al., dealing with low-dimensional conservative systems. Their analysis focuses on series expansions of invariant manifolds, whose intersection points serve to provide the location of a wide range of chaotic domains. In particular, they find the domain of convergence of Moser type series around unstable periodic orbits and are able to obtain analytic expressions for asymptotic manifolds as well as homoclinic and heteroclinic orbits. They apply their techniques first to the simple hyperbolic Hénon map, and then turn to problems of astronomical interest related to the spiral structure of barred-spiral galaxies.

Next, G. Voyatzis reviews the topic of multi - planetary systems and examines their possible formation due to the trapping of a third planet by a resonance with two others, caused by the action of dissipative forces. Migration of planetary systems caused by the action of dissipative forces may lead the planets to be trapped in a resonance. He studies the conditions and dynamics of resonance trapping to find out whether resonant capture ends up in a long-term stable planetary configuration. The author reports on applications of these results to specific low order resonances in systems consisting of two and three planets focusing on the formation of stable configurations.

M. Robnik points out in his paper the importance of time-dependent Hamiltonians in modeling the interaction of conservative systems with their environment. He begins by reviewing some recent results on time-dependent, parametrically driven, one-dimensional linear oscillators, for which the complete analysis can be performed, using e.g. the linear WKB method. Then he points out that, in a class of parametrically driven nonlinear oscillators with homogeneous power law potentials, extensive numerical studies and applications of a nonlinear WKB method have shown that the energy is not conserved. In particular, he discusses new results demonstrating that close to the adiabatic regime an initial Dirac delta distribution spreads in time by slow variations. On the other hand, under sudden parametric kicks, the energy changes according to the conservation of an adiabatic invariant, and follows distributions that are very different from the linear case.

As E. Gerlach et al. point out in their paper, disordered, discrete Nonlinear Schrödinger equations (DDNLS) are very popular models for investigating the diffusion of wave packets in one dimensional Hamiltonian lattices, under the combined presence of nonlinearity and disorder. In this work, the authors present several methods utilizing the techniques of symplectic integration to solve numerically the equations of motion of a DDNLS that possesses a few hundreds of degrees of freedom. 
They discuss their results and compare the efficiency of different symplectic integration techniques, suggesting that the most suitable ones for long time integration of such multi-degree-of-freedom Hamiltonian systems are those that are based on a three part split of the systems Hamiltonian.

The next paper by C.E. Cantos et al. is motivated by modeling flock behavior in the motion of autonomous vehicles (see also the article by van der Weele and Kanellopoulos in this section). The authors study the transient behavior of a large array of $\mathrm{N}$ coupled linear damped harmonic oscillators (or agents), upon perturbing the first element of the array. Imposing a number of conjectures, they use an asymptotic theory for large $\mathrm{N}$ to show that minimizing the transients renders the system asymptotically stable, approaching a state where all agents have the same velocity. The system may be stable, but the transient perturbations can still grow exponentially large with $\mathrm{N}$, leading to a phenomenon called flock instability.

In the paper by K.v.d. Weele and G. Kanellopoulos, the authors construct a model of traffic on a ring road, which demonstrates that density car waves can move either as small-amplitude disturbances in the same direction, or as high-amplitude waves (or "jams") in the opposite direction. The model exploits an analogy between granular and traffic flow to show the spontaneous generation of density waves, explain their stability properties, and determine the density level at which the waves change direction. Of course, as the authors point out, cars are "self-driven" entities in which drivers do their best to avoid collisions, whereas granular particles are inanimate objects that bump into each other all the time. To be sure, the mesoscopic view adopted in the paper (i.e. that individual driving styles are more or less averaged out within each cell) bridges some of these differences. Still, the adopted approach can be viewed as a first step in building more realistic traffic flow models that will bear visible similarities with conscious behavior expected by active drivers.

In the next paper, Basios et al. examine the phenomenon of aggregation, which appears in many complex systems in the form of variation of temporal and/or spatial density of individuals (in biology) or particles (in physics), as a result of pattern formation and self organization [9]. They examine first the rules of aggregation in problems of biology, where individuals belonging to two sub-groups are attracted to each other, while having to choose between two options. The authors develop a mathematical model based on previous experimental studies and point out certain similarities that exist between aggregation in their biological problems and pattern formation in such important physical processes as crystallization, non-standard nucleation, self-assembly and particle growth.

Tsigkri-DeSmedt et al. model the transmission of electric signals in neuron networks using the Leaky Integrate-and-Fire model for the neuron potential, while neurons are coupled in a ring architecture. When nonlocal coupling is assumed, multichimera states emerge whose multiplicity depends on the parameters of the system and in particular on the neuron refractory period. The idea of fractal coupling is introduced which originates from the hierarchical topology of networks formed by neuron axons which transmit the electrical signals in the brain. This hierarchical topology in the coupling induces novel complex spatial and temporal structures, such as nested chimera states and transitions between multi-chimera states with different multiplicities. These novel structures arise as combined effects of the nonlinear dynamics with the hierarchical coupling.

In the final article of the Papers I section, M. Choi et al. propose two different approaches to coarse-graining large heterogeneous networks illustrated via neuronal network examples. They employ methods of uncertainty quantification in systems with multiple uncertain parameters heterogeneously distributed among the network nodes, demonstrate that their approach can successfully deal with structural as well as intrinsic heterogeneities, and use dimension reduction techniques to analyze the dynamics. In the first network, the ANalysis-Of-VAriance (ANOVA) method is used to 
expand the instantaneous states of the neurons in polynomials whose time-dependent coefficients are the variables of a reduced model. In the second network, the state of each neuron is again expressed as a sum of polynomials and a novel generalized Polynomial Chaos (gPC) approach is used. These approaches show promise in accelerating large scale network simulations as well as coarse-grained fixed point, periodic solution and stability analysis.

\section{The section of papers II}

In the first paper of this section, by Or Alus et al., recent new results are presented concerning the distribution of rotation numbers of boundary cycles of the Hénon map, connected with the phenomenon of "stickiness" of orbits on the boundaries of regular motion in conservative dynamical systems. The authors report on the probability distributions of the continued fraction expansion elements of such cycles, showing that the elements are not bounded, contrary to previous conjectures. Probabilities of flux ratios between adjacent sites on the binary Markov tree are calculated, which support the validity of the Meiss and Ott Markov tree model describing the dynamics in these domains.

Next, in the paper by I. Mylonas et al., the authors study nonlinear wave propagation in periodic media and investigate the stability and internal modes of onedimensional gap solitons located between the Bloch bands of a modified nonlinear Schrödinger equation in the presence of a sinusoidal potential and weak nonlocality. They prove that two soliton families bifurcate from every Bloch-band edge under selffocusing or self-defocusing nonlinearity, and one of these is always unstable. They also analyze oscillatory instabilities and symmetry breaking bifurcations, demonstrating that their analytical results are in excellent agreement with numerical computations.

The article by C.E. Cantos et al. belongs to an area of research that aims to develop mathematical methods to study the phenomenon of flocking. One particularly useful application would be to enable cars to achieve coherent motion on a highway, even under dense traffic conditions. To this end, the authors consider a system of coupled oscillators on the circle as vehicles, whose acceleration depends on being informed about the relative positions and velocities of their neighbors. After deriving necessary and sufficient conditions for asymptotic stability, the authors obtain expressions for the phase velocity of propagation of velocity disturbances through the system. Then they examine the problem of controlling the growth of disturbances as the number of vehicles becomes large, since transients may still grow exponentially with the number of cars, even in the case of asymptotic stability. As the spectrum of the associated linear operator does not help one recognize the problem, the authors develop an alternative approach, approximating asymptotically stable solutions by traveling wave signals.

In the next paper, Kanellopoulos and van der Weele study longitudinal density waves in granular media, and present a novel way by which these waves can organize themselves, resembling the succession of "merlons" and "crenels" on the walls of a medieval castle. These are observed in transport systems consisting of $\mathrm{K}$ connected compartments through which agitated granular matter flows in one direction. In a regime of medium bias the flow takes the form of waves whose particle density, inside any compartment, oscillates around its average value. For $\mathrm{K}$ odd, this value is the same for all compartments, but, for $\mathrm{K}$ even, the symmetry is spontaneously broken and the average value is alternatingly higher and lower in successive compartments giving rise to a "merlon" wave.

Next, V. Basios explores the emergence of the curious phenomenon of stochastic resonance in biologically motivated chaotic models, namely the Rössler and the 
Thomas-Rössler models. By introducing a probabilistic switch which stochastically modifies the system parameters he shows that transitions occur to orderly behaviour when the amplitude of the noise increases. Then, J. Hizanidis et al. discuss chimera states and synchronisation phenomena in quantum mechanical systems and in particular in SQUID metamaterials. They consider a one-dimensional linear array of SQUIDs coupled together through dipole-dipole magnetic forces. When the interactions become nonlocal the authors observe multistability phenomena and single and multichimera states as well as synchronisation desynchronisation transitions.

Dynamical phenomena connected with drug release are studied in the paper by Christidi and Kalosakas to explore the influence of the boundaries in the dynamics of the release. Using both analytical approximations and Monte Carlo simulations they show that drug release from spherical or slab devises causes the motion of the drug molecules to deviate from Fickian diffusion approaching a stretched exponential distribution.

A network model based on the exactly known connectome of the nervous system of the C.elegans is used in the work of Antonopoulos et al. to study synchronization phenomena and exchange of information in living organisms. Since the precise dynamics of the C. elegans organism is not known, each node is modeled by a 4th order system of Hindmarsh-Rose equations. The C. elegans nervous system is shown by two techniques to be divided into (6) communities (modules) and nodes within each module are linearly coupled, while nonlinear coupling schemes are applied between modules. This modular network shows that integrated information attains maximum values when either all modules are highly synchronized or in mixed, chimera states of highly synchronized and desynchronized communities.

In the paper by de Sanctis and Gattone, a new statistical method is proposed to analyze 2 dimensional complex shapes, in which landmark data are represented by a bivariate Gaussian distribution. The authors first use the Fisher-Rao metric of Information Geometry to show that the statistical manifold in the parameter space of a family of probability distributions possesses a Riemannian metric. This allows them to reconstruct the evolution of a sequence of observed shapes by computing the geodesic path of the corresponding distributions. This path can then be used for shape predictions in specific applications of biological and medical interest.

Holiday and Kevrekidis use the equation-free modeling approach to analyze a dynamically evolving multigraph, coupling short intervals of direct dynamic network simulation with appropriately-defined lifting and restriction operators, mapping the detailed network description to suitable macroscopic (coarse-grained) variables. Two coarse graining techniques are employed to enable the acceleration of direct simulations, and linear (principal component analysis, PCA) as well as nonlinear (diffusion map) methods are used to achieve dimensional reduction and determine good macroscopic variables for an efficient and accurate description of the system's time evolution.

The aim of the authors of the next paper, M. Boudourides and S. Lenis, is to address the problem of decomposing a whole network into a minimal number of ego centered subnetworks, as the members of a minimum dominating set of the network. As they point out, to do this, one needs to use graph theoretic concepts like adjacency and minimum dominating sets to compare all possible decompositions of the network, which is a very arduous task. For this purpose, the authors develop a heuristic based on the partition of the set of nodes of a graph into three subsets, the always dominant vertices, the possible dominant vertices and the never dominant vertices, and apply their techniques to analyze six empirical social networks, using algorithms implemented in the Python - based SageMath. Software.

The final paper of the Papers II section, by Y. Sakamoto and I. Vodenska, investigates the Japanese banking crisis of the late 1990s using a simple 
mathematical model based on the properties of a bi-partite bank-asset network, which allows the authors to simulate the crisis through an analytic solution of their model. They are thus able to study the robustness of the banking system, as well as reveal aggregate time dynamics of bank asset prices throughout the banking crisis. The results disclose fundamental properties of asset growth, and enable the estimation of the selling pressure for each asset type, through a Cascading Failure Model (CFM). Since the shock of consecutive bank failures spread to the rest of Asia shortly following the collapse of many Japanese banks it is important to understand this crisis as the cause of a cascading risk phenomenon.

Tassos Bountis, Jeff Johnson, Astero Povata and George Tsironis

Guest Editors

March 31, 2016

We wish to thank the Editors of the European Physics Journal - Special Topics for their excellent cooperation in helping us publish this volume.

\section{References}

1. G. Nicolis, I. Prigogine, Exploring Complexity (Freeman, New York, 1989)

2. M.M. Waldrop, Complexity: The Emerging Science at the Edge of Order and Chaos (Simon and Schuster, 1993)

3. G. Nicolis, C. Nicolis, Foundations of Complex Systems (World Scientic, Singapore, 2007)

4. Two Special Issues of the International Journal of Bifurcation and Chaos: Vol. 16, Issue: (6), June 2006 and Vol. 16(7), July 2006) of Proceedings of International Conference Complexity in Science and Society, Patras and Olympia, 1426 July, 2004

5. A. Pikovsky, M.G. Rosenblum, J. Kurths, Synchronization, A Universal Concept in Nonlinear Sciences (Cambridge University Press, Cambridge, 2001)

6. M. Newman, A.L. Barabasi, D.J. Watts, The structure and Dynamics of Networks (Princeton University Press, 2006)

7. C. Tsallis, Introduction to Nonextensive Statistical Mechanics: Approaching a Complex World (Springer, New York, 2009)

8. T. Bountis, H. Skokos, Complex Hamiltonian Dynamics (Synergetic Series, Springer, 2012)

9. E. Schöll, S.H.L. Klapp, P. Hövel (eds.), Control of Self-Organizing Nonlinear Systems (Springer, Berlin, 2016) 